\title{
Substance Use Dose
}

National Cancer Institute

\section{Source}

National Cancer Institute. Substance Use Dose. NCI Thesaurus. Code C83416.

The amount of substance that a subject has used. 